\title{
Acute Disseminated Encephalomyelitis after Oral Therapy with Herbal Extracts: A Case Report
}

\author{
Bahar Kaymakamzade ${ }^{1}$, Rana Karabudak ${ }^{2}$, Aslı Tuncer Kurne², Gülay Nurlu² \\ ${ }^{1}$ Department of Neurology, Near East University School of Medicine, Nicosia, Cyprus \\ ${ }^{2}$ Department of Neurology, Hacettepe University School of Medicine, Ankara, Turkey
}

Background: Acute disseminated encephalomyelitis (ADEM) is a rare demyelinating disease of the central nervous system, commonly attributed to infections or vaccinations. Toxic or allergenic compounds can also trigger a response in the immune system and may cause demyelination. We present a case with ADEM after using oral herbal medications.

Case Report: A 25 year-old male developed bilateral central facial palsy and severe quadriparesis after taking herbal drugs (containing echinacea and many other herbal ingredients) for two weeks. He had used the extract to increase his potency and reproductivity. He had no past history of recent immunization or viral infection. The clinical findings, cerebrospinal fluid (CSF) analysis and brain magnetic resonance imaging (MRI) were compatible with ADEM. The neurological findings were improved after seven doses of pulse methylprednisolone treatment. To our knowledge, this is the third report in the literature that links herbal therapy and demyelinating disease.

Conclusion: Most of the ADEM cases related to herbal therapy in the literature similarly used echinacea. It is our opinion that other ingredients of the herbal extract used by our case, besides echinacea, could have the potential to cause a trigger in the immune system. Further studies are needed to clarify the immunological effects of different kinds of herbal compounds, as well as the effects of different parts of the plants and the results of various dosages. Moreover, ingredients should also be tested for toxicity, adverse effects and drug interactions.

Keywords: Acute disseminated encephalomyelitis, autoimmunity, demyelination, Echinacea, herbal medicine, phytotherapy
Acute disseminated encephalomyelitis (ADEM) is typically a monophasic or rarely a multiphasic immune-mediated inflammatory disorder. Some of the patients may be at risk for the development of Multiple Sclerosis. It affects children more than adults. It is commonly attributed to a post-infectious etiology and less frequently to a post-vaccination complication. There is a time interval of a few days up to four weeks between the causative insult and the onset of neurological symptoms. Herein, we report a case with ADEM after taking oral herbal medication.

\section{CASE PRESENTATION}

A 25 year-old male was admitted to our hospital with chief complaint of severe weakness in both upper and lower extremities that had started two days ago. The weakness and numbness had started from the left upper extremity and spread to other extremities. He did not have a past history of recent immunization or viral infection; however, he had been taking two kinds of herbal tablets three times a day for the last two weeks. The drugs contained Carthamus tincto-

This study was presented at the Joint Congress of European Neurology, 31 May - 3 June 2014, İstanbul, Turkey.

Address for Correspondence: Dr. Bahar Kaymakamzade, Department of Neurology, Near East University School of Medicine, Nicosia, Cyprus

Phone: +905338878087_ e-mail: bkaymakamzade@yahoo.com

Received: 18 May $2014 \quad$ Accepted: 19 June 2015 D DOI: 10.5152/balkanmedj.2016.140420

Available at www.balkanmedicaljournal.org 

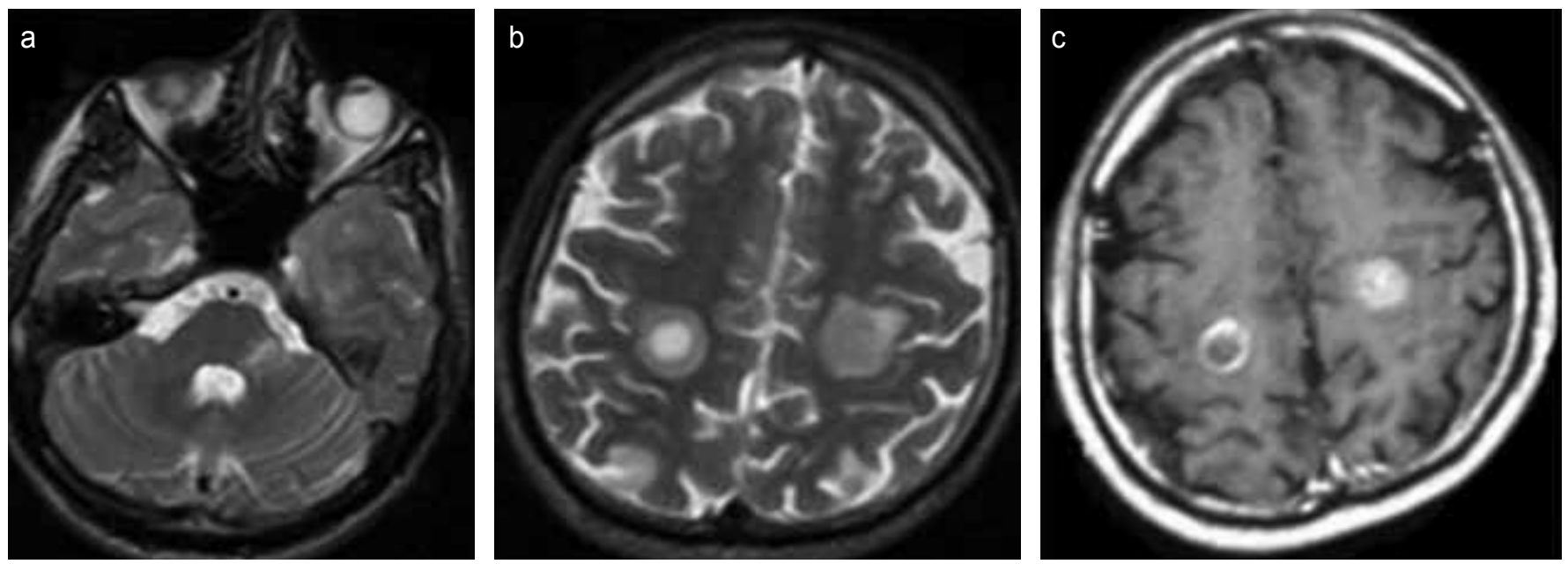

FIG. 1. a-c. MRI showed T2 hyperintense ovoid lesions in left pontocerebellar area (a), right corona radiata (b) and bilateral parietal areas with contrast enhancement (c).

rius, pollen, stinging nettle, fennelflower, ginger, galingale, Myristica Frangrans, vitamin C, Beta glucan and echinacea. He used the extract to increase his potency and reproductivity. The vital signs, including body temperature, blood pressure, respiratory rate and heart rate, were all in the normal range. He was alert, fully-oriented and cooperative. Cranial nerve examination revealed bilateral central facial palsy. Motor system assessment showed grade 3 weakness in the proximal and grade 1 in the distal right upper extremity. The left upper extremity was completely plegic. Mild weakness (grade 4) was detected in the right lower extremity while the proximal of the left extremity had mild (grade 4) and the distal of the left extremity had moderate (grade 3) weakness. He described left sided hemihypoestesia and the deep tendon reflexes were hyperactive, but he did not have a pathological reflex. The cerebellar functions could not be evaluated due to the patient's weakness. The magnetic resonance imaging (MRI) (Signa, GE Medical Systems, Wisconsin, United States of America) of the brain showed ovoid T2 hyperintense lesions in the left pontocerebellar area, the right corona radiata and bilateral asymmetry in parietal lobes with contrast enhancement (Fig 1). Cervical spinal MRI was normal. Blood studies, including complete blood count, electrolytes, liver, renal, thyroid function tests and sedimentation rate, were all normal. Vasculitis markers including antinuclear antibodies (ANA), anti-ds DNA, anti-cardiolipin IgM, IgG antibodies, anti-phosphatidylserine IgM, IgG antibodies, perinuclear anti-neutrophil cytoplasmic antibodies (p-ANCA), cytoplasmic anti-neutrophil cytoplasmic antibodies (cANCA), anti-la and anti-ro antibodies and rheumatoid factor were within normal limits. He tested negative for HIV. Cerebrospinal fluid (CSF) protein and glucose levels, and IgG index were also within normal limits. Seven doses of pulse methyl prednisolone treatment (with a dosage of 1gr/day) was started. The motor weakness started to improve on the third day of the treatment and he was able to walk and use his left upper extremity at the end of the pulse steroid treatment. Three weeks later, the patient was discharged from hospital without an apparent deficit. Brain MRI was performed using 0.3-T open-magnet MRI system (AIRIS, Hitachi Medical Systems America Inc.; Ohio, USA) one month after the treatment. T2 hyperintense lesions persisted while contrast enhancement disappeared and no additional lesion was detected (Figure 2). Follow-up of the patient has been carried out for four years without any exacerbation.

\section{DISCUSSION}

Although the pathogenesis is not fully understood, ADEM appears to be an autoimmune disorder of the central nervous system (CNS) that is triggered by an environmental stimulus. ADEM may be due to increased vascular permeability and congestion in the CNS, perhaps triggered by circulating immune complexes or other humoral factors that develop after the appearance of a foreign antigen (1). The clinical findings, CSF analysis and MRI results of our patient were compatible with ADEM. There was a close temporal relation between herbal medication and the onset of neurological symptoms. The absence of antecedent infection or immunization and the temporal concomitance led us to think of a relationship between ADEM and herbal extracts in our patient. In addition, the cited case reports supported our consideration. Two cases with ADEM after parenteral therapy and one patient with acute demyelinating disease after oral therapy with herbal extracts have been reported in the lit- 

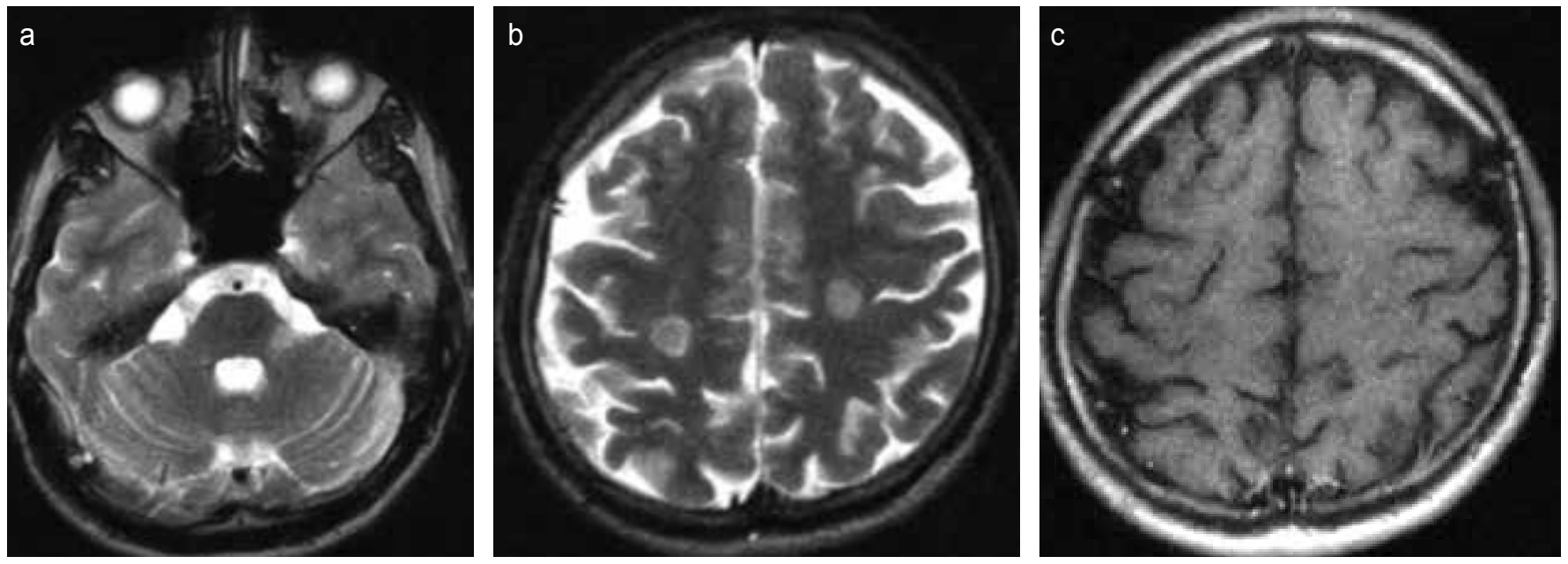

FIG. 2. a-c. MRI showed persistence of T2 hyperintense lesions in left pontocerebellar area (a), and right corona radiata (b), while contrast enhancement disappeared in parietal areas (c).

erature $(2,3)$. To the best of our knowledge, this is the third report that links herbal therapy and demyelinating disease. Schwarz et al. (2) reported two patients with ADEM after parenteral therapy with herbal extracts in 2000. Both patients had no history of viral infection or immunization. The first case used herbal extract (echinacea, aconitum and lancesis) subcutaneously twice a week to prophylactically support the immune system. Fourteen days after the last dose, neurologic symptoms appeared and symptoms rapidly improved after a five-day methyprednisolone $(500 \mathrm{mg} /$ day) treatment. The other patient used plant extract for four weeks by intramuscular injections to lose weight. It was a saline solution (Adonis vernalis, calcium carbonicum, capsium, Cascara sagrada, Fucus vesiculosus, graphites, phylolacca and sulfur). She was treated with methylprednisolone $(500 \mathrm{mg} /$ day) for five days and then continued with oral administration for four weeks. The patient gradually recovered and neurologic examination returned to normal (2). In 2011, Kostianovsky et al. (3) published the first case with acute demyelinating disease after oral therapy with herbal extracts (Echinacea purpurea, Uncaria tomentosa, Tabebulia avellanedae and Plantago maritime). She had started the treatment two weeks before the onset of symptoms for cervical pain. After admission to hospital, her neurologic examination and brain lesions progressively worsened. She was treated with 3 g methylprednisolone intravenously (i.v.), oral steroids, five plasma exchange sessions and $120 \mathrm{~g}$ immunoglobulin i.v. However, the symptoms did not improve and she was finally discharged with severe neurologic deficits. Although CSF and MRI findings were compatible with ADEM in this patient, because of the unremitting, progressive and persistent severe sequel, the authors preferred to describe it as an acute or unclassified immune-mediated demyelinating event.
Herbal medications have been used for centuries and are regarded by the population as natural and harmless. Although there is an increasing knowledge in the literature demonstrating that they are not always safe, they have still been widely used for different purposes, such as for the prevention or treatment of common cold and minor infections (4). Herbal compounds potentially contain ingredients that can provoke a response in the immune system and may induce demyelination in the central nervous system. Together with our case, three out of the four patients in the literature had used extracts that contain echinacea. There are many reports of immune reactions associated with echinacea, such as anaphylaxis, asthma, maculopapular rash and autoimmune hepatitis $(5,6)$. The information from the literature published to date is limited and the mechanism modifying the immune system is not well-known. Echinacea may have immunostimulant effects, such as increasing cytokine and immunoglobulin (Ig) production $(7,8)$. Unsaturated N-alkylamide lipids, the main constituent of echinacea, have been suggested to play a role as potential anti-inflammatory and proinflammatory effects $(9,10)$. It was reported that echinacea alkylamides modulate tumor necrosis factor alpha (TNF- $\alpha$ ) gene expression via cannabinoid receptor type 2 (CB2) and multiple signal transduction pathways (9). Some experimental studies have shown that TNF- $\alpha$ expression was higher in animals who received diet containing echinacea, while some showed lower TNF- $\alpha$ production in echinacea-fed animals $(8,11)$. Yamada et al. (8) observed increased cytokine (IL2 and interferon gamma) production from $\mathrm{T}$ helper 1 cells with dietary echinacea and significant increases in the serum IgG level and all Ig production in the spleen. It was stated that the effect of alkylamides on the immune system depends on the stimulus applied, drug concentration used and degree 
of unsaturation of the lipophilic tail of the specific alkylamide. It was determined that different results of the studies are related to the different kinds and parts of this plant (10). The second case of Schwarz et al. (2) developed ADEM, although she did not use echinacea. Some of the ingredients she used could have immunostimulatory properties, such as Cascara sagrada and capsicum, which were reported to induce occupational respiratory allergic diseases and irritant contact dermatitis, respectively $(12,13)$. It is in our consideration that other ingredients of the herbal extract used by our case besides echinacea could have the potential to trigger the immune system. In light of this information, further studies are needed to clarify the immunological effects of different herbal compounds, as well as the effects of different parts of the plants and the results of various dosages. Moreover, ingredients should also be tested for toxicity, adverse effects and drug interactions.

In conclusion, we aimed to make physicians aware of such cases and highlight the fact that the contents of herbal drugs might induce autoimmunity.

\section{Ethics Committee Approval: N/A.}

Informed Consent: Written informed consent was obtained from patient who participated in this study.

Peer-review: Externally peer-reviewed.

Author contributions: Concept - B.K., R.K., A.T., G.N.; Design B.K., R.K., A.T., G.N.; Supervision - B.K., R.K., G.N.; Resource B.K., R.K., A.T., G.N.; Materials - B.K., R.K., G.N.; Data Collection and/or Processing - B.K., R.K., A.T., G.N.; Analysis and/or Interpretation - B.K., R.K., A.T., G.N.; Literature Search - B.K., R.K., A.T., G.N.; Writing B.K., R.K.; Critical Reviews - B.K., R.K.

Acknowledgements: Authors would like to thank Dr. Hülya Efetürk for improving the use of English in the manuscript.

Conflict of Interest: No conflict of interest was declared by the authors.

Financial Disclosure: The authors declared that this study has received no financial support.

\section{REFERENCES}

1. Rudick RA, Eskin TA. Enhanced vascular permeability in acute disseminated vasculomyelinopathy. Ann Neurol 1981;9:621. [CrossRef]

2. Schwarz S, Knauth M, Schwab S, Walter-Sack I, Bonmann E, Storch-Hagenlocher B. Acute disseminated encephalomyelitis after parenteral therapy with herbal extracts: a report of two cases. J Neurol Neurosurg Psychiatry 2000;69:516-8. [CrossRef]

3. Kostianovsky A, Maskin P, Noriega MM, Soler C, Bonelli I, Riley CS, et al. Acute demyelinating disease after oral therapy with herbal extracts. Case Rep Neurol 2011;3:141-6. [CrossRef]

4. Singh D, Gupta R, Saraf SA. Herbs-are they safe enough? an overview. Crit Rev Food Sci Nutr 2012;52:876-98. [CrossRef]

5. Mullins RJ, Heddle R. Adverse reactions associated with echinacea: the Australian experience. Ann Allergy Asthma Immunol 2002;88:42-51. [CrossRef]

6. Kocaman O, Hulagu S, Senturk O. Echinacea-induced severe acute hepatitis with features of cho-lestatic autoimmune hepatitis. Eur J Intern Med 2008; 19:148. [CrossRef]

7. Burger RA, Torres AR, Warren R, Caldwell VD, Hughes BG. Echinacea-induced cytokine production by human macrophages. Int J Immunopharmacol 1997;19:371-9. [CrossRef]

8. Yamada K, Hung P, Park TK, Park PJ, Lim BO. A comparison of the immunostimulatory effects of the medicinal herbs Echinacea, Ashwagandha and Brahmi. J Ethnopharmacol 2011;137:231-5. [CrossRef]

9. Gertsch J, Schoop R, Kuenzle U, Suter A. Echinacea alkylamides modulate TNF-alpha gene expression via cannabinoid receptor CB2 and multiple signal transduction pathways. FEBS Lett 2004;577:563-9. [CrossRef]

10. Raduner S, Majewska A, Chen JZ, Xie XQ, Hamon J, Faller B, et al. Alkylamides from Echinacea are a new class of cannabinomimetics. Cannabinoid type 2 receptor-dependent and -independent immunomodulatory effects. J Biol Chem 2006;281:14192206. [CrossRef]

11. Uluışık D, Keskin E. Effects of ginseng and echinacea on cytokine mRNA expression in rats. ScientificWorldJournal 2012;2012:942025. [CrossRef]

12. Giavina-Bianchi PF Jr, Castro FF, Machado ML, Duarte AJ. Occupational respiratory allergic disease induced by Passiflora alata and Rhamnus purshiana. Ann Allergy Asthma Immunol 1997;79:449-54. [CrossRef]

13. Otang WM, Grierson DS, Afolayan AJ. A survey of plants responsible for causing irritant contact dermatitis in the Amathole district, eastern cape, south Africa. J Ethnopharmacol 2014;157:274-84. [CrossRef] 